\title{
Lossy Image Compression Using Wavelet Transform, Polynomial Prediction And Block Truncation Coding
}

\author{
${ }^{1}$ dr. Ghadah Al-Khafaji, ${ }^{2}$ rafaa Ismael Yahya \\ Dept. of Computer Science, Baghdad University, College of Science. \\ Dept. of Computer Science, Al-Mustansiriyah University, College of Science.
}

\begin{abstract}
In this paper, a lossy image compression is introduced, it based on utilizing three techniques of wavelet, polynomial prediction and block truncation coding, in which each technique exploited in away according to redundancy presents. The test results shown are promising performance in terms of higher compression performance achieved with lower noticeable error or degradation.
\end{abstract}

\section{Introduction}

Image compression of lossy based have become an increasingly intensive research area, due to importance to our daily visual media applications, including TV, video film, the internet etc, which basically based on losing some unrecognized or unwanted information, and managing non-noticeable distortion quality changes is traded off against high compression ratios. The extremely useful and well known lossy international standards, JPEG and JPEG 2000, based on transform coding that utilizes discrete cosine transform (DCT) and discrete wavelet transform (DWT), respectively, characterized by highly compression efficiency without visual degradation - that is to say that the result is still quite visually pleasing [1-4]. On the other hand, various lossy techniques available still under development such as vector quantizer, block truncation and fractal, based on exploiting the spatial coding efficiently. Reviews of various lossy techniques can be found in [5-9]. In this paper a hybrid efficient image compression technique is introduced based on exploited the transform coding of wavelet transform and spatial coding of polynomial prediction coding and block truncation. The rest of the paper is organized as follows, section 2 contains comprehensive clarification of the proposed system; the results of the proposed system is given in section 3 .

\section{The Proposed System}

The implementation of the hybrid lossy image compression system of multiresolution wavelet based along with polynomial and block truncation is explained in the following steps, the layout of the proposed system is illustrated in Figure 1:

Step 1: Load the original uncompressed gray image $I$ of size $N \times N$.

Step 2: Apply two successive wavelet transform, starts by decomposing the image $I$ into first layer (layer ${ }_{1}$ ) of four quadrants $\left(L L_{l}, L H_{l}, H L_{l}\right.$ and $\left.H H_{l}\right)$ each of size $(N / 2 \times N / 2)$, then subsequently decompose the first layer detail sub bands of $L H_{l}$ and $H L_{l}$ into second layers each of four quadrants of size $(N / 4 \times N / 4)$. The techniques simply based on utilizing the wavelet transform more than once, by using the details subband of the preceding layer. The resultant, quadrants images with various resolution and details obtained (i.e., $L L_{l}$ and $H H_{l}$ of layer ${ }_{1}$, while $L L_{2}, L H_{2}, H L_{2}$ and $H H_{2}$ of layer ${ }_{2}$ of $L H_{1}$ and $H L_{1}$ ), each utilized the redundancy embedded in different way to improve the compression performance.

Step 3: For the approximation subband of the first layer $\left(L L_{l}\right)$ that resembles the original image $I$ of size $(N / 2 \times N / 2)$, the polynomial prediction model of linear base adopted such as:

1- Partition the $\left(L L_{l}\right)$ into non-overlapping blocks of squared fixed sized regions of $n \times n$, and compute the coefficients according to the equations $(1,2 \& 3)[10-11]$.

$$
a_{0}=\frac{1}{n \times n} \sum_{i=0}^{n-1} \sum_{j=0}^{n-1} L L_{1}(i, j) \ldots \ldots \ldots . . .(1) \quad a_{1}=\frac{\sum_{i=0}^{n-1} \sum_{j=0}^{n-1} L L_{1}(i, j) \times\left(j-x_{c}\right)}{\sum_{i=0}^{n-1} \sum_{j=0}^{n-1}\left(j-x_{c}\right)^{2}} \ldots \ldots .(2)
$$$$
a_{2}=\frac{\sum_{i=0}^{n-1} \sum_{j=0}^{n-1} L L_{1}(i, j) \times\left(i-y_{c}\right)}{\sum_{i=0}^{n-1} \sum_{j=0}^{n-1}\left(i-y_{c}\right)^{2}} \ldots \ldots . .(3)
$$

Where $L L_{l}(i, j)$ is the first layer approximation sub-band of original image block of size $(n \times n)$ and $x c=y c=\frac{n-1}{2}$...... ...(4)

Each block of size $n \times n$ represented by three coefficients $\left(a_{0}, a_{l} \& a_{2}\right)$ corresponds to the mean, ratio of the pixel to the distance from the center in $\mathrm{x}$ and $\mathrm{y}$ direction, respectively. 
2-Quantize the coefficients using the scalar uniform quantizer. The coefficients quantized with different quantization steps according to its importance, where the $a_{0}$ quantized with higher quantization level to keep the image information that represented by mean perceived as much as possible, while the $a_{1} \& a_{2}$ quantized with the same less quantization step compared to $a_{0}$. The dequantized required to reconstruct the approximated coefficients, the quantizer/dequantizer as shown in equations 5-7.

$a_{0} Q=\operatorname{round}\left(\frac{a_{0}}{Q S_{a 0}}\right) \rightarrow a_{0} D=a_{0} Q \times Q S_{a 0} \ldots \ldots . .(5)$
$a_{1} Q=\operatorname{round}\left(\frac{a_{1}}{Q S_{a_{\text {coff }}}}\right) \rightarrow a_{1} D=a_{1} Q \times Q S_{a_{\text {coff }}} \ldots \ldots \ldots . . .(6)$
$a_{2} Q=\operatorname{round}\left(\frac{a_{2}}{Q S_{a_{\text {coff }}}}\right) \rightarrow a_{2} D=a_{2} Q \times Q S_{a_{\text {coff }}} \ldots \ldots . . .(7)$

Where $Q S_{a 0}$ quantization step of $a_{0}$ coefficient and $Q S_{a_{c o f f}}$ quantization step of both $a_{1} \& a_{2}$ coefficients.

3-Create the predicted image value $\widetilde{I}$ using the dequantized polynomial coefficients of each block representation:

$\tilde{L} L_{1}=a_{0} D+a_{1} D\left(j-x_{c}\right)+a_{2} D\left(i-y_{c}\right) \ldots \ldots \ldots \ldots \ldots \ldots .(8)$

The predicted image $\tilde{I}$ resemble the original image but with less accuracy due to the prediction principle.

4- Find the residual as the difference or error between the predicted and original one.

$R(i, j)=L L_{1}(i, j)-\tilde{L} L_{1}(i, j) \ldots \ldots .(9)$

5- Quantize the residual image, as discussed previously in (2) using the uniform scalar quantizer, such that:

$R Q=\operatorname{round}\left(\frac{R}{Q S_{R}}\right) \ldots \ldots \ldots \ldots \ldots \ldots . . .(10)$

Where $Q S_{R}$ quantization step of residual image.

Step 4: For the detail subband of the first layer $\left(H H_{l}\right)$ that represents the source of compression, in which it is not rich with data [12-13]. Block truncation coding of $n_{s} \times n_{s}$ (i.e., $n_{s}<n$ ) efficiently used, due to utilizing the one-bit quantizer (i.e., binary quantizer of two values of 0 and 1 ) scheme that basically based on the statistical moments; extensive details of the block truncation coding techniques can be found in [14-16].

Step 5: For the second layers sub bands, the system used the polynomial prediction techniques mentioned above used with the block truncation coding, such as:

1- For the approximation subband ( $\left.L L_{2}\right)$ of $L H_{l}$ and $H L_{l}$, the polynomial prediction techniques adopted (step 3), but with small block size than layer1 that equal to $n_{s} \times n_{s}\left(n_{s}<n\right)$.

2- For the detail sub bands $\left(\mathrm{LH}_{2}, \mathrm{HL}_{2} \& H \mathrm{H}_{2}\right)$, the block truncation coding used (step 4) of block size equal to $n_{s} \times n_{s}\left(n_{s}<n\right)$

Step 6: Encodes the compressed information, where for binary images resultant from the block truncation the run length coding utilized efficiently followed by LZW, whereas the quantized residual along with information composed of coefficients $\left(a_{0}, a_{1} \& a_{2}\right)$ and the moments (mean \& standard deviation of block truncation) the LZW followed by Huffman coding exploited.

Step 7: To reconstruct the compressed (decoded) image, the reversed steps followed:

1 - Decodes the encoded compressed information to reconstruct the values.

2- Dequantized the residual image, by multiplying by the residual quantization step.

$R D=R Q \times Q S_{R}$

3- The approximation subband reconstructed using:

$L L_{1}(i, j)=R D(i, j)+\tilde{L} L_{1}(i, j)$..

4- The second layer sub bands utilized subsequently to reconstruct the first layer then the first layer information used to reconstruct the image. 


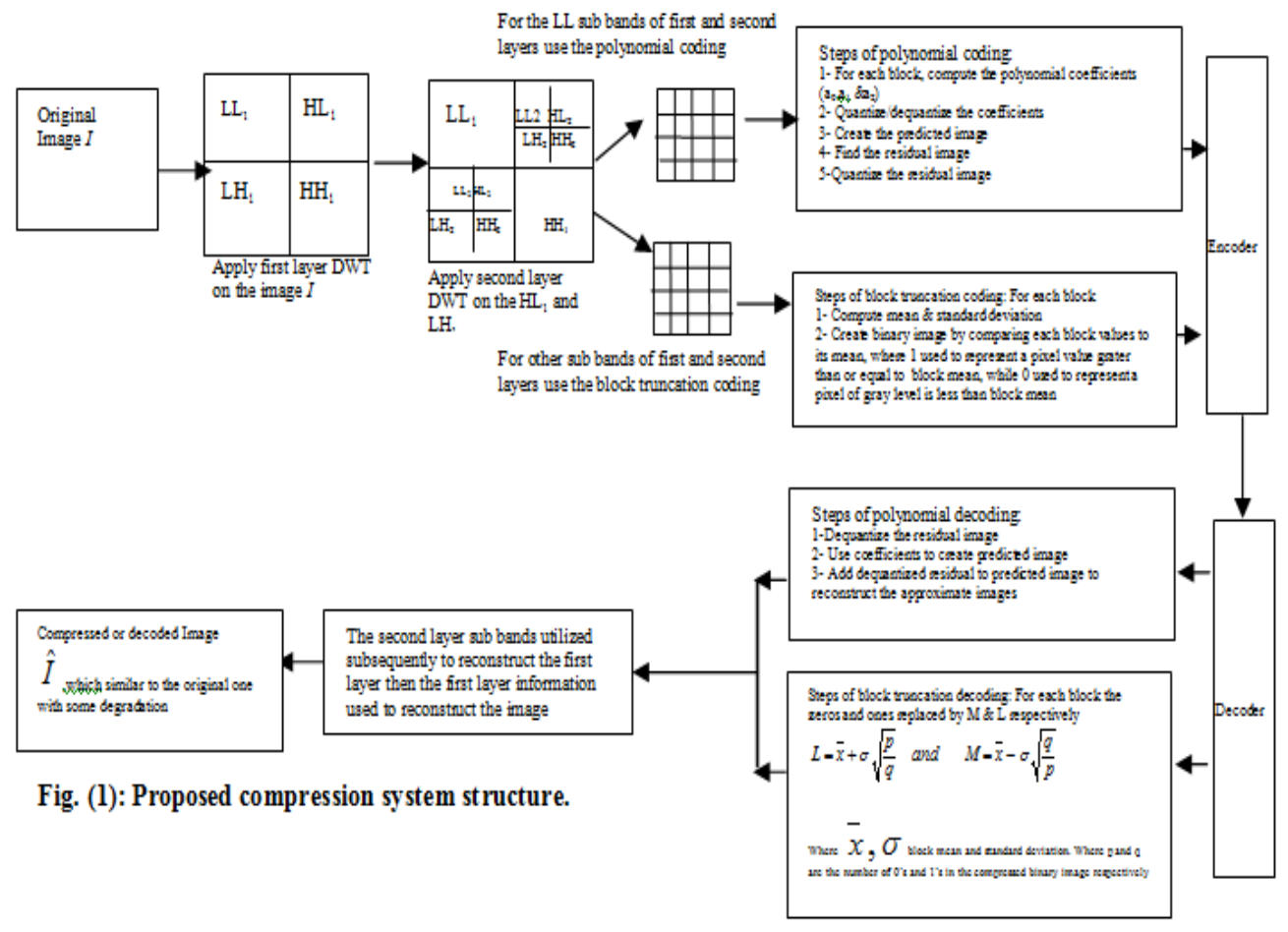

\section{Experimental and Results}

Basically for the lossy type the compression evaluation and image quality (i.e., fidelity criteria) used as tools to test the performance of the proposed system. The images used in the experiments are of different variation of details (see Figure 2 for an over view), all the images are square $(256 \times 256)$ gray images. The tests have been performed using Haar DWT of two layers, the block sizes adopted $\{4 \times 4\}$ and $\{2 \times 2\}$ for polynomial prediction and block truncation coding respectively, and different quantization steps are used to quantize the coefficients and residual images in first and second layers. The experimental result listed in Table (1), using the objective quantities measures, based on Compression Ratio and Normalized Root Mean Square Error (see equations $13 \& 14$ respectively). The examples of decompressed tested images are shown in Figure 3.

Comp.Ratio $=\frac{\text { Original } \operatorname{Image~Size}}{\text { CompressedSize }}$

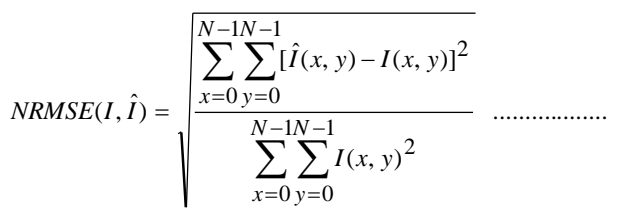

It is clear that the quantization step inversely affected both the compression ratio and quality, where as a small quantization step a high compression attainted with low quality, and as the quantization step gets bigger the compression decrease with high quality. Also the results illustrates that the quantization steps of approximation subband of layer1 $\left(L L_{l}\right)$ that implicitly implies the coefficients and residual, higher than the approximation sub bands of layer2 of $L H_{1}$ and $H L_{l}$; in other words high quantization steps applied to layer ${ }_{1}$ compared to layer ${ }_{2}$ to preserve the information as much as possible. As well as, the higher quantization step of $a_{0}$ in both layers are utilized to keep the image details by preserving the mean efficiently. Certainly, the quantization step of residual images affected the appearance of the image (i.e., image quality), due to limitation in modelling efficiency; in other words the image information that can not be predicted accurately, actually found in the residual, which leads to noticeably small error of quantization coefficients compared to the quantization of the residual.

In general, the results vary according to the block size of two layer techniques. This means for polynomial coding and block truncation coding two block sizes adopted, also for the prediction techniques of approximation subband $\left(L L_{l}\right)$ the block size of first layer bigger than the second layer. 
Lastly, using the hybrid techniques of transformation based along with prediction and block truncation increase the system performance in terms of compression ratio \& quality of images, where results vary according to image features or nature.

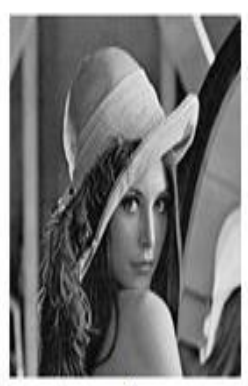

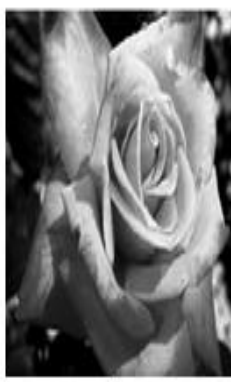

b

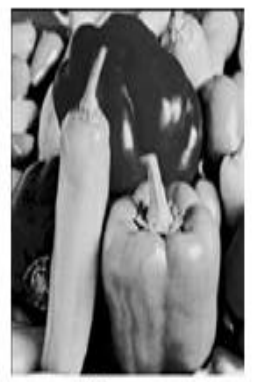

C

Fig.(2): Overview of the tested images (a) Lena image, (b) Rose image and (c) Paper image all images of size $256 \times 256$, gray scale images.

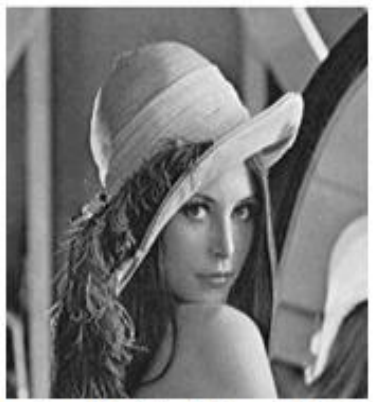

Qant. Step of LL subband of layer $=16,8,8,32$ Qant. Step of LL aubband of laver $=8,4,416$ $\mathrm{CR}=19.1308$

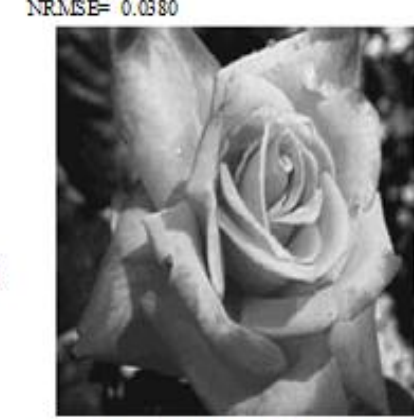

Qent. Step of LL subband of layer $=16,8,8,32$ Qant. Step of LL subbend of layer $=8,4,4,16$ $\mathrm{CR}=19.8678$

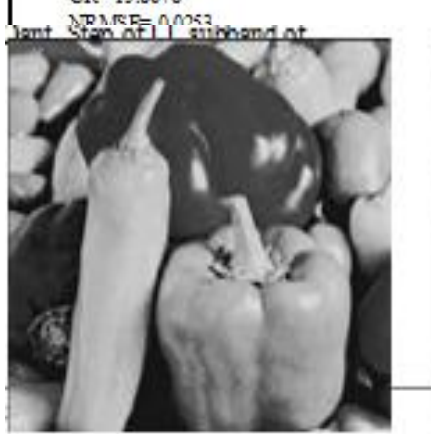

Qant Stepp of LL subband of layer $=16,8,8,32$

Oant. Steo of LL subband of

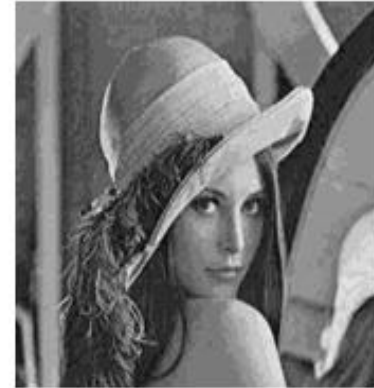

Qant. Step of LL subband of layer, $=8,4,4,16$ Qant. Step of LL subband of layer $=4,2,2,16$ $\mathrm{CR}=41.4489$

$\mathrm{NRMSE}=0.0616$

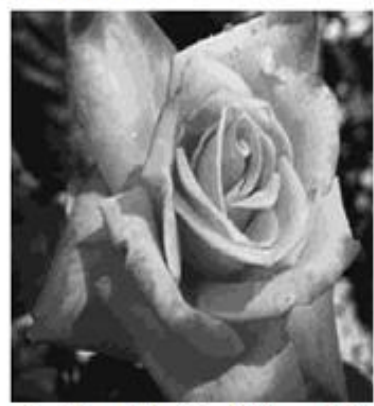

Oant Step of LL auboand of laver $=8.44,16$ Qant. Step of LL auboand of layer $=4,2,21$ $\mathrm{CR}=40.5988$

NRMSE $=0.041$

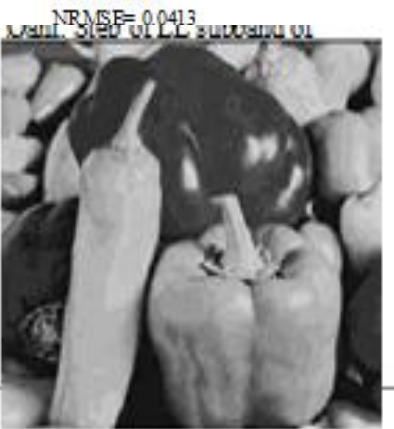

Qant Step of LL subound of layer $=8,4,4,16$

Oant. Step of LL subbend of

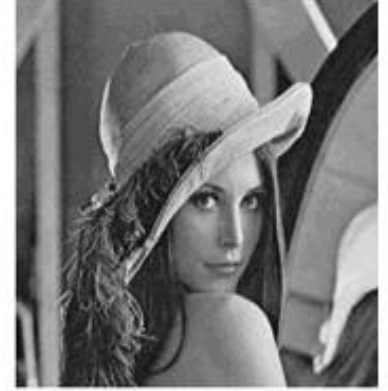

Qant. Step of LL subband of layer $=16,2,2,3$ Qant. Step of LL subband of layer $=4,2,2,16$ $\mathrm{CR}=40.1174$

NRMSE $=0.0429$

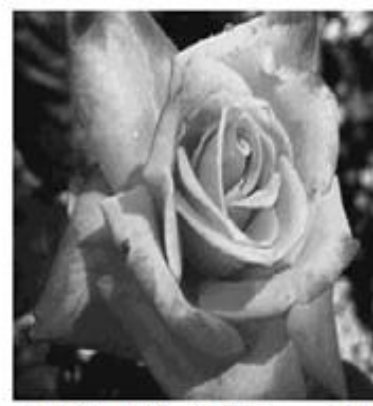

Qant. Step of LL subband of layer $=16,22,32$ Qant Step of LL subbend of layer: $=4,2,216$ $\mathrm{CR}=323317$

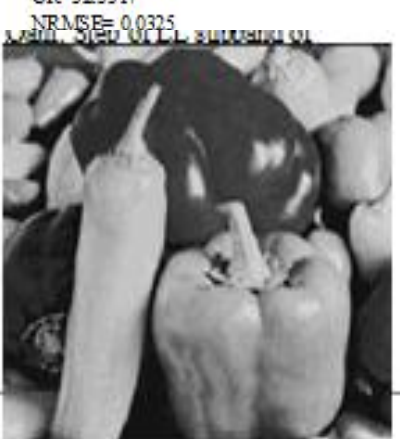

Qint Step of LL subbend of layer $=16,22,32$

Oant. Step of LL subbond of

Fig. (3): Example of compressed tested images (a) Lena image, (b) Rose image and (c) Paper image 
Table 1: The performance of the proposed system on the tested images using different quantization steps values for the residual images and the coefficients of layer ${ }_{1}$ and layer $_{2}$

\begin{tabular}{|c|c|c|c|c|c|c|c|c|c|c|c|c|c|c|}
\hline \multirow[t]{2}{*}{$\begin{array}{l}\text { Test } \\
\text { Images }\end{array}$} & \multicolumn{4}{|c|}{$\begin{array}{l}L L_{1} \text { Quantization Steps } \\
\text { of coefficients and } \\
\text { Residual image }\end{array}$} & \multicolumn{4}{|c|}{$\begin{array}{l}L L_{2} \text { of } L H_{1} \text { Quantization } \\
\text { Steps of coefficients and } \\
\text { Residual image }\end{array}$} & \multicolumn{4}{|c|}{$\begin{array}{l}L L_{2} \text { of } H L_{1} \text { Quantization } \\
\text { Steps of coefficients and } \\
\text { Residual image }\end{array}$} & \multicolumn{2}{|c|}{$\begin{array}{l}\text { Block size }\{4 \times 4\} \text { of } \\
\text { polynomial approximation \& } \\
\text { Block size }\{2 \times 2\} \text { of block } \\
\text { truncation coding. }\end{array}$} \\
\hline & $\mathrm{a}_{0}$ & $\mathrm{a}_{1}$ & $\mathrm{a}_{2}$ & Res & $a_{0}$ & $a_{1}$ & $a_{2}$ & Res & $\mathrm{a}_{0}$ & $a_{1}$ & $a_{2}$ & Res & Comp. Ratio & NRMSE \\
\hline \multirow[t]{6}{*}{ Lena } & 16 & 8 & 8 & 16 & 8 & 4 & 4 & 16 & 8 & 4 & 4 & 16 & 32.74 & 0.0504 \\
\hline & 16 & 8 & 8 & 32 & 8 & 4 & 4 & 32 & 8 & 4 & 4 & 32 & 11.83 & 0.0372 \\
\hline & 16 & 4 & 4 & 32 & 8 & 2 & 2 & 16 & 8 & 2 & 2 & 16 & 28.20 & 0.0419 \\
\hline & 16 & 2 & 2 & 32 & 4 & 2 & 2 & 32 & 4 & 2 & 2 & 32 & 19.42 & 0.0417 \\
\hline & 8 & 4 & 4 & 16 & 4 & 2 & 2 & 32 & 4 & 2 & 2 & 32 & 34.90 & 0.0607 \\
\hline & 8 & 2 & 2 & 32 & 4 & 2 & 2 & 16 & 4 & 2 & 2 & 16 & 35.33 & 0.0434 \\
\hline \multirow[t]{6}{*}{ Rose } & 16 & 8 & 8 & 16 & 8 & 4 & 4 & 16 & 8 & 4 & 4 & 16 & 31.92 & 0.0413 \\
\hline & 16 & 8 & 8 & 32 & 8 & 4 & 4 & 32 & 8 & 4 & 4 & 32 & 11.36 & 0.0248 \\
\hline & 16 & 4 & 4 & 32 & 8 & 2 & 2 & 16 & 8 & 2 & 2 & 16 & 22.18 & 0.0256 \\
\hline & 16 & 2 & 2 & 32 & 4 & 2 & 2 & 32 & 4 & 2 & 2 & 32 & 22.66 & 0.0314 \\
\hline & 8 & 4 & 4 & 16 & 4 & 2 & 2 & 32 & 4 & 2 & 2 & 32 & 35.78 & 0.0404 \\
\hline & 8 & 2 & 2 & 32 & 4 & 2 & 2 & 16 & 4 & 2 & 2 & 16 & 39.28 & 0.0327 \\
\hline \multirow[t]{6}{*}{ Paper } & 16 & 8 & 8 & 16 & 8 & 4 & 4 & 16 & 8 & 4 & 4 & 16 & 39.15 & 0.0390 \\
\hline & 16 & 8 & 8 & 32 & 8 & 4 & 4 & 32 & 8 & 4 & 4 & 32 & 8.93 & 0.0262 \\
\hline & 16 & 4 & 4 & 32 & 8 & 2 & 2 & 16 & 8 & 2 & 2 & 16 & 24.13 & 0.0315 \\
\hline & 16 & 2 & 2 & 32 & 4 & 2 & 2 & 32 & 4 & 2 & 2 & 32 & 17.28 & 0.0312 \\
\hline & 8 & 4 & 4 & 16 & 4 & 2 & 2 & 32 & 4 & 2 & 2 & 32 & 35.11 & 0.0462 \\
\hline & 8 & 2 & 2 & 32 & 4 & 2 & 2 & 16 & 4 & 2 & 2 & 16 & 32.95 & 0.0321 \\
\hline
\end{tabular}

\section{References}

[1]. Wallace, G. K. 1992. The JPEG Still Picture Compression Standard. IEEE Transactions on Image Processing, 38(1), xviii-xxxiv.

[2]. Christopoulos, C., Skodras, A. and Ebrahimi, T. 2000. The JPEG2000 Still Image XOSING System: an Overview. IEEE Transactions on Consumer Electronics, 46(4), 1103-1127.

[3]. Taubman, D. and Marcellin, M. 2004. JPEG2000: Image Compression Fundamentals, Standards \& Practices. Kluwer Academic Publishers.

[4]. Acharya, T. and Tsai, P. 2004. JPEG2000 Standard for Image Compression: Concepts, Algorithms and VLSI Architectures. John Wiley \& Sons, Hoboken, New Jersey.

[5]. Jabbar, S. and Panchikkil, S. 2012. Lossy Image Compression Scheme based on Transforms- A Literature Review on Medical Images. International Journal of Advanced Information Technology (IJAIT), 2(6), 25-31.

[6]. Kuppusamy, K. and Ilackiya, R. 2013. Fractal Image Compression \& Algorithmic Techniques. International Journal of Computer \& Organization Trends (IJCOT), 3(4),141-145.

[7]. Mathur, G. Mathur, R. and Kumar, M. 2014. A Comparative Study of Various Lossy Image Compression. International Conference on Emerging Trends of Research in Applied Sciences and Computational Techniques, ETRASCT 14 Conference Proceedings, 165 168 .

[8]. Arora, K. and Shukla, M. 2014. A Comprehensive Review of Image Compression Techniques. International Journal of Computer Science and Information Technologies (IJCSIT), 5(2),1169-1172.

[9]. Amin, B. and Amrutbhai, P.2014. Vector Quantization based Lossy Image Compression using Wavelets- A Review. International Journal of Innovative in Science, Engineering and Technology (IJIRSET), 3(3), 10517-10523

[10]. George, L. E. and Sultan, B. 2011. Image Compression Based on Wavelet, Polynomial and Quadtree. Journal of Applied Computer Science \& Mathematics (JACSM), 11(5), 15-20.

[11]. Ghadah, Al-K. 2013. Image Compression based on Quadtree and Polynomial. International Journal of Computer Applications (IJCAs), 76(3),31-37.

[12]. Ranjan, A. and Chandra, M. 2010. Image Compression based on Wavelet and Quantization with Optimal Huffman Code. International Journal of Computer Applications (IJCAs), 5(2), 6-9.

[13]. Rajesh, K., Gangwar, S. and Vikram, H. 2012. Study and Analysis of Wavelet based Image Compression Techniques. International Journal of Engineering, Science and Technology (IJEST), 4(1), 1-7.

[14]. Pasi, F. and Olli, N. 1995. Block Truncation Coding with Entropy Coding. IEEE Transactions on Communications, 43(2/3/4), 16771685 .

[15]. Somasundaram, K., and Vimala, S. 2010. Efficient Block Truncation Coding. International Journal on Computer Science and Engineering (IJCSE), 2(6), 2163-2166.

[16]. Doaa, M., and Fatma, A. 2011. Image Compression Using Block Truncation Coding. Cyber Journals: Multidisciplinary Journals in Science and Technology, Journal of Selected Areas in Telecommunications (JSAT), February edition, 9-13. 